\title{
Projeto Design Condensado: Definição de 'Modelo Sociocultural' para Sistemas de Visualização Dinâmica de Dados que Estimulem a Geração de Novos Conhecimentos sobre a História do Design
}

\author{
Condensed Design Project: the definition of a 'social-cultural model' for dynamic data visualization \\ systems which stimulate knowledge building in design history
}

\author{
Katia Akemi Omine \\ Universidade Anhembi Morumbi \\ kakemi.omine@gmail.com \\ José Neto de Faria \\ Universidade Anhembi Morumbi \\ josenetodesigner@yahoo.com.br
}

Karine Itao Palos

Universidade Anhembi Morumbi

karineipa@hotmail.com

\begin{abstract}
The 'Condensed Design Project' aims to discuss and reflect on how the definition of an 'activity inducer', by describing and characterizing 'social-cultural models', can be used in the development of dynamic data visualization systems. The aim is to discuss and try to understand how the profile of the 'activity inducer', routines, scenario and context impact the navigation, interaction, research and learning processes in design history. Thus, the knowledge building process is strictly conditioned to the way the relation between the 'visualization system' and the 'activity inducer' is promoted, considering the technological limitations and the individual's predisposition.
\end{abstract}

Keywords: Data visualization; Design history; Usage context, Experience; Knowledge.

\section{Visualização Sociedade e Conhecimento}

O desenvolvimento de sistemas de visualização dinâmica de dados dependente estritamente do estudo e da descrição do 'modelo sociocultural' que define o processo perceptivo e cognitivo dos 'Indutores de Atividade'. Assim, com o intuito de implantar um sistema de visualização dinâmica de dados para promover a pesquisa, o ensino e o aprendizado da história do design, além de se estudar os princípios e as técnicas de visualização de dados, pesquisou-se o perfil sociocultural da população brasileira, restringindo o estudo aos aspectos relacionados a condições de vida e à escolaridade, para depois relacionar com o perfil perceptivo e cognitivo do 'Indutor de Atividade'. Pois, a capacidade do sistema de instigar distintos processos de significação, depende da capacidade de promover, no 'Indutor de Atividade', o enfrentamento da realidade e elaborar rotinas que potencializem a percepção, a seleção, a organização, a categorização, a análise, a interpretação e a narração de 'acontecimentos históricos' de moda a gerar a formação de novos conhecimentos.

As informações processadas podem ser organizadas ou escalonadas em três níveis distintos: dados primários e ou brutos, as chamadas sensações e ruídos; dados elaborados e ou organizados, informação ditas com sentido; e por fim, informação analisada, interpretada, sintetizada e validada, ou o considerado conhecimento consolidado (Turban; Potter; Rainer, 2007). A formação do conhecimento se dá a partir do momento em que uma informação é percebida, absorvida, processada, reformulada e posteriormente sintetizada, com base em informações e conhecimentos vivenciados pelo 'Indutor de Atividade' (Weibel apud Miranda, 1998). Assim, o 'Indutor de Atividade' permanece sempre preso a cenários, a rotinas e a contextos de trocas de informações (W3schools, 2013).

\section{Metodologia}

Os procedimentos metodológicos (Akker; Bannan; Kelluy; Nieveen; Plomp, 2010) adotados na definição do 'modelo sociocultural' para o sistema de visualização dinâmica de dados foram divididos em oito etapas: pesquisa referencial sobre as condições de vida e a escolaridade da população brasileira entre 18 e 24 anos de idade, presentes no Censo IBGE 2011 (Instituto Brasileiro de Geografia e Estatística, 2013); definição do 'modelo sociocultural' com base nos dados estatísticas coletados das cinco regiões do Brasil, sul, sudeste, centro-oeste, nordeste, norte; descrição de cada uma das personas as quais caracterizem os 'Indutores de Atividade' brasileiros (Rosenfeld; Morville, 2002; Santaella, 1998); teste com mock-up's nos locais em que os 'Indutores de Atividade' costumam usar dispositivos digitais (Cooper, 1998; Rosa; Moraes, 2012a; Rosa; Moraes, 2012a); ajustes e correções do 'modelo 
sociocultural'; definição de cenários de uso e de personas ou 'Indutores de Atividade'; teste de comportamento de uso, produção de 'Vídeos Conceituais'; e por fim, ajustes e correções do 'modelo sociocultural'.

\section{Percepção e Cognição}

A percepção consiste na capacidade que tem o 'Indutor de Atividade' de perceber algo exterior. A percepção é seletiva, dividida em diferentes planos nem sempre em foco, perceber é estar diante de algo e conseguir vê-lo e compreendê-lo. As sensações são definidas pelo 'Indutor de Atividade', exposto a conjuntos de objetos, que são captados como arrepios, cores, sons, toques, odores e gostos, características que estão condicionadas a eficiência e as limitações dos sentidos (Gibson, 1950).

Contudo, no modelo sensitivo proposto por Damásio (2004, p.116) explica que existe uma estrutura mais complexa que define o processamento dos sentimentos e construção da percepção, baseada: em sinais sensitivos transmitidos ao cérebro; quimicamente (correntes sanguínea) ou de forma neural (dividido em meio interno, vísceras, músculos estriados e sistema vestibular; contato mecânico, tato; contato químico, olfato e paladar; e telessensação, visão e audição).

As condições para existirem os sentimentos e as percepções são as seguintes: primeira condição, "ter não só um corpo, mas também meios para representar esse corpo dentro de si mesmo"; segunda condição, ter um sistema nervoso "capaz de mapear as estruturas do corpo e os seus diversos estados e ser capaz também de transformar os padrões neurais desses mapas em padrões mentais"; terceira condição, ter o conteúdo desses sentimentos reconhecidos pelo organismo, pois a "consciência é também uma necessidade básica para a ocorrência do sentimento", lembrando que os "dispositivos fisiológicos do sentimento fazem parte dos processos que dão origem à consciência"; quarta condição, ter o organismo um cérebro que sente, e ao mesmo tempo, capaz de criar "os estados corporais que evocam sentimentos", resultado da reação do organismo a objetos e situações, pois somente o cérebro pode produz "os mapas neurais do estado do corpo", mas antes que esses mapas possam ser produzidos, corpo e cérebro devem construir "os estados emocionais do corpo", cujo seu "mapeamento permite os sentidos" (Damásio, 2004, p.118-120).

Desta forma, todo o processo perceptivo o qual capta as informações é diferente, não é frente ao processo cognitivo que não só percebe as informações, mas mantém um estado de sensações que estabelece o que é percebido. Não sendo, então somente um processo de decodificação, codificação, recodificação, estocagem, recuperação e utilização, de um conjunto claramente definido de informações (Nunes; Giraffa apud Tarouco; Santos, 2007).
A identificação de um determinado objeto dá início ao processo de transmissão e sintetização da informação, a qual promove a percepção de estados os quais podem gerar novos conhecimentos. Entende-se por conhecimento uma mistura fluida de raciocínios lógicos, experiências, valores, informação contextual e conhecimento especializado o qual fornece o âmbito necessário para a avaliação e a inclusão de novas experiências e informações (Davenport; Prusak apud Bonsiepe, 2013). Assim, o conhecimento significa mais que ter acesso à informação, revelase como um conceito de conhecimento hermenêutico, que se fundamenta na seleção, esclarecimento, interpretação, compreensão, tradução, e que também envolve processamento da informação, mas o qual busca diferenciar-se do conceito de conhecimento puramente instrumental, que visa à ação eficiente (Bonsiepe, 2013). A aquisição de um determinado conhecimento pode ser dita válida, quando o conjunto de informações pode ser sintetizado tendo algum tipo de significância no repertório do 'Indutor de Atividades', a aquisição é armazenada em uma cadeia de conhecimentos relevantes, a qual posteriormente pode ser utilizada ou consultada.

\section{Personas, Rotinas, Cenário e Contexto}

A definição de personas é uma metodologia de pesquisa usada na definição do perfil do 'indutor de atividade', que busca ajudar a entender as características do usuário, as rotinas de trabalho, o cenário de uso e o contexto de aplicação e uso de sistemas digitais interativos.

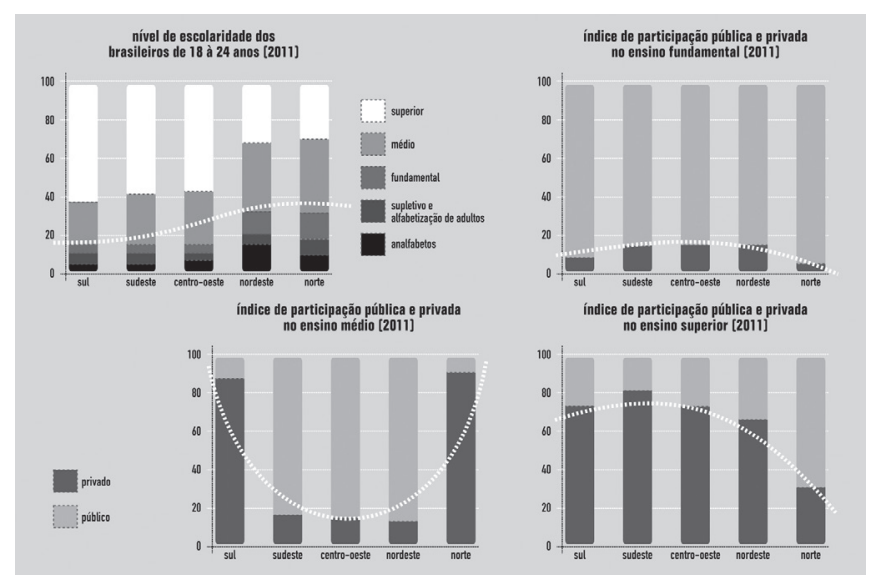

Figura 1: Índice de Escolaridade - Escolaridade da população brasileira registrada no Censo 2011 (IBGE, 2013).

Assim, usaram-se os dados estatísticos da população brasileira sobre educação e precariedades sociais, do Censo 2011, do Instituto Brasileiro de Geografia e Estatística - IBGE (2013), para montarem-se as personas de 18 anos a 24 anos que caracterizariam o público-alvo do projeto. Como apresenta as figuras (Fig.1) (Fig.2), pode-se notar que existe uma diferença acentuada entre as regiões sul e sudeste em comparação com as regiões nordeste e norte, as quais apresentam um déficit maior 
em escolaridade, seguridade social, condições de moradia, acesso a serviços básicos e a uma renda digna.

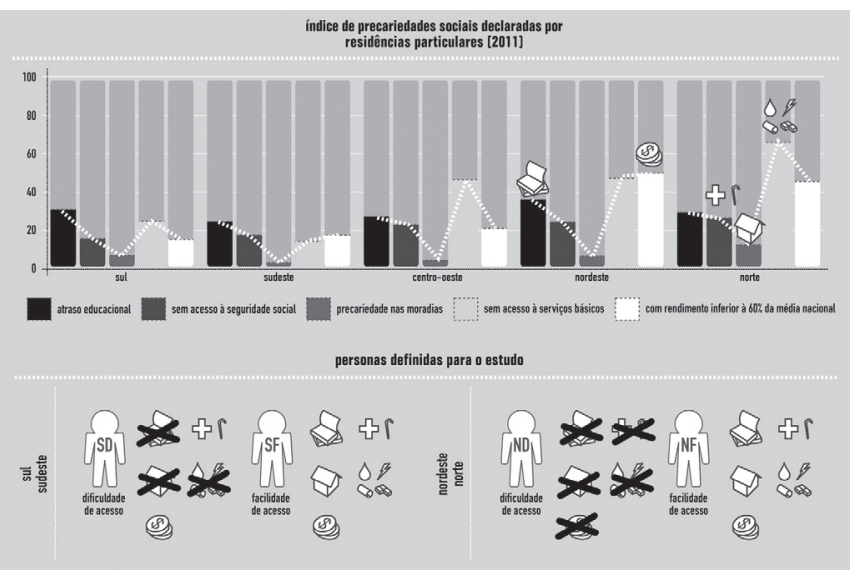

Figura 2: Índice de Precariedade - Condição de vida da população brasileira registrada no Censo 2011 (IBGE, 2013).

Observando-se a proximidades destes parâmetros, decidiram-se agrupar as cinco regiões brasileiras em dois grandes grupos: grupo GS, sul, sudeste e centro-oeste; e grupo GN, nordeste e norte. Em cada grupo, definiu-se duas personas as quais caracterizam os estados extremos da população: persona SD, 'sul-sudeste com restrições de acesso'; persona SF, 'sul-sudeste com acesso'; persona ND, 'nordeste-norte com restrições de acesso'; e persona $\mathrm{NF}$, 'nordeste-norte com acesso'.

Persona SD, 'sul-sudeste com restrições de acesso', predomínio da população com: baixa renda de origem negra e branca; atraso médio na escolaridade com taxa de analfabetismo de 4,90\% da população; $18,70 \%$ sem acesso a seguridade social; moradia precária ou modesta; com falta de pelo menos um dos serviços básicos; adeptos da cultura urbana das grandes cidades; acesso restrito a roupas de marca e a supérfluos; e a residência quando possui um Computador Pessoal com acesso à internet, é de segunda mão e compartilhado entre os moradores.

Persona SF, 'sul-sudeste com acesso', predomínio da população com: média e alta renda de origem branca; com acesso ao ensino superior de $65,40 \%$ da população; acesso a seguridade social; moradia confortável e prática; com acesso a todos os serviços básicos; adeptos de uma cultura urbana cosmopolita, integrada as grandes capitais do mundo; com acesso a roupas de marca e a supérfluos; e a residência possui mais de um Computador Portátil, Computador Pessoal ou dispositivo móvel, como iPad, com acesso à Internet e geralmente os dispositivos não são compartilhados entre os moradores.

Persona ND, 'nordeste-norte com restrições de acesso', predomínio da população com: condição de miséria ou baixa renda de origem negra, índia e branca; atraso extremo ou médio na escolaridade com taxa de analfabetismo de $16,90 \%$ da população; $28,30 \%$ sem acesso à seguridade social; moradia extremamente precária ou modesta; com falta de mais de dois serviços básicos; inclusos na cultura regional rural ou urbana; sem acesso a roupas de marca e a supérfluos; e com grande maioria da população sem Computador Pessoal e sem acesso à Internet, geralmente, dependendo de instituições públicas, organizações não governamentais ou Lan Houses.

Persona NF, 'nordeste-norte com acesso' predomínio da população com: média e alta renda de origem branca; com acesso ao ensino superior de $35,80 \%$ da população; acesso a seguridade social; moradia confortável e tradicional; com acesso a todos os serviços básicos; adeptos de uma cultura urbana integrada as grandes capitais do país; com acesso a roupas de marca e a supérfluos; e a residência possui mais de um Computador Portátil e Computador Pessoal, com acesso à Internet e geralmente os dispositivos não são compartilhados entre os moradores.

\section{Mock-up's e 'Vídeos Conceituais'}

No desenvolvimento do sistema de visualização dinâmica de dados foram utilizados nos testes: Mock-up's, com uma amostra de 12 pessoas, com características próximas as definidas para cada uma das personas selecionadas, nos locais onde costumam usar seus dispositivos digitais; e 'Vídeos Conceituais', quatro vídeos, um para cada persona, nos quais o roteiro contemplou aspectos de uso do sistema, preferências por plataformas, cenários de uso do sistema e cotidiano de rotinas do 'Indutor de Atividade'.

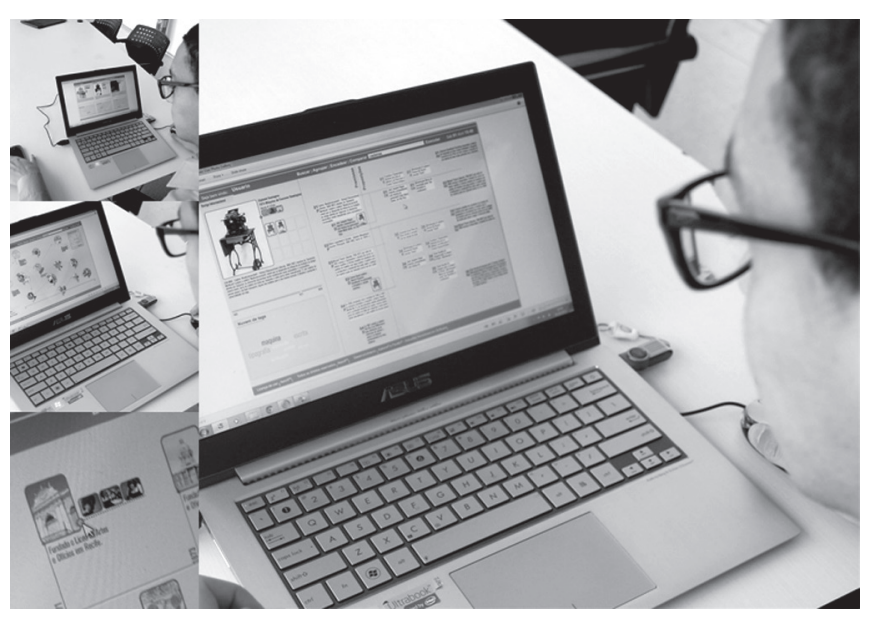

Figura 3: Mock-up’s - Aplicação de teste com a Persona NF.

Os Mock-up's, com o auxílio de Checklist's, foram utilizados a fim de testar a usabilidade do sistema, organização da informação, integração e eficácia das interfaces, mecânicas de navegação, e, por finalmente, aspectos psicológicos da interatividade, considerando o 'modelo sociocultural' definido para cada uma das personas (Fig.3) (Fig.4). Os 'Vídeos Conceituais', com o auxílio de Checklist's, foram utilizados para verificar as qualidades do sistema inserido nas rotinas, nos cenários e no contexto de uso, do mesmo modo, considerando o 'modelo sociocultural' definido para cada uma das personas (Fig.5) (Fig.6). 


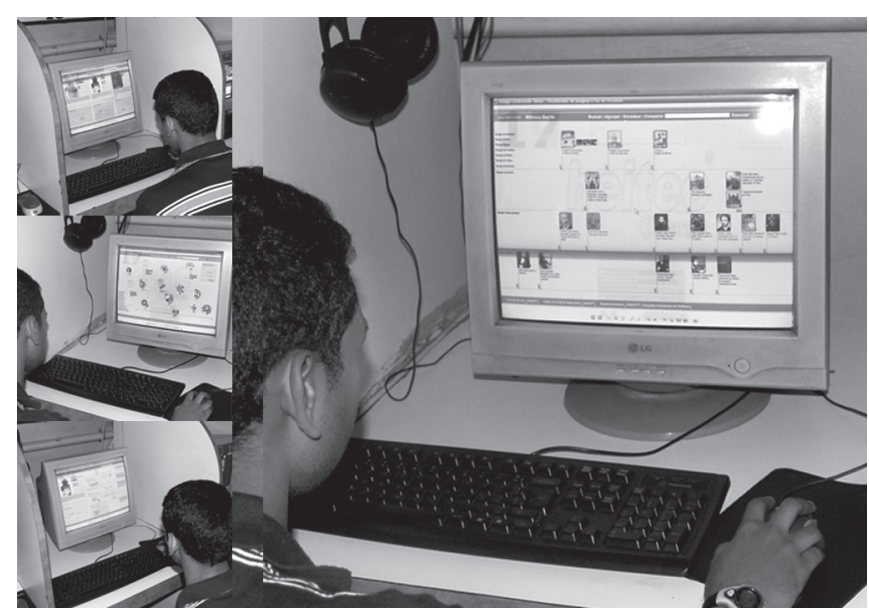

Figura 4: Mock-up’s - Aplicação de teste com a Persona ND.

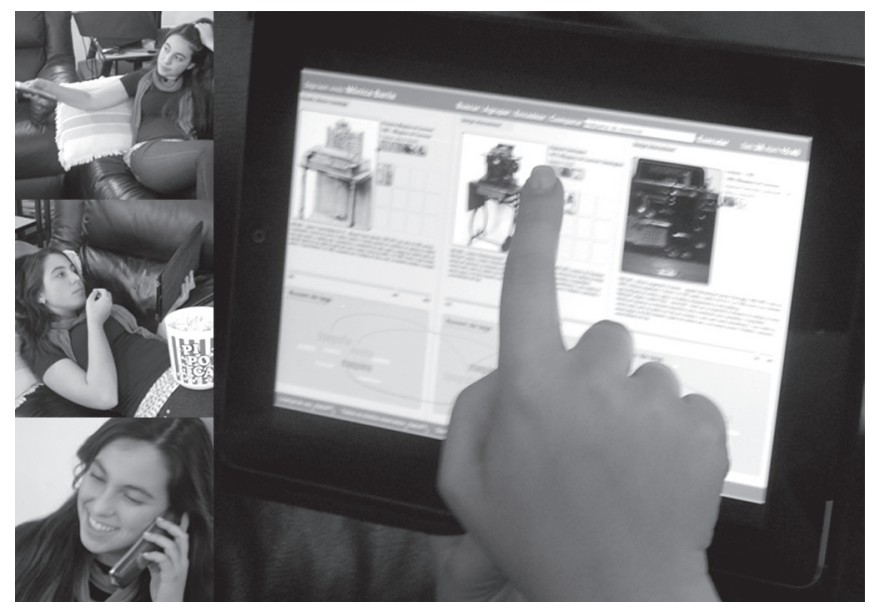

Figura 5: 'Vídeos Conceituais' - Inserção do sistema nas rotinas e cenários de uso da Persona SF.

\section{Visualização / Cotidiano}

O perfil definido no 'modelo sociocultural' permitiu ter indícios de que a escolaridade e as condições de vida do Brasileiro, infelizmente influenciam o desenvolvimento e o desempenho perceptivo e cognitivo. Contudo, devem ser desconsideradas as diferenças causadas por aspectos e valores regionais, os quais determinam predisposições perceptivas e cognitivas não dependentes das condições de vida.

O ambiente e as rotinas de trabalho, definidas pelo estilo de vida de cada persona, é responsável por uma conjugação de fatores que potencializam ou não o aprendizado. Durante a aplicação dos testes com os Mock-up's, nas observações registradas nos Checklist's, ficou evidente, na percepção dos aplicadores, que ambientes em que o 'Indutor de Atividade' não tem o controle sobre o fluxo de atividades, existe uma propensão maior para à dispersão, dificultando o uso do sistema de visualização de dados. Do mesmo modo, em que rotinas de trabalho mais intensas promovem a dispersão, pela necessidade de não se focar numa atividade, a fim de terminar o mais rápido possível cada atividade, para que se possa começar mais um dia.

Assim, pode-se dizer que a interpretação das visualizações dos 'fatos históricos' não fica condicionada as qualidades conceituais e técnicas do sistema, depende também, da consideração e da ponderação da influência dos aspectos perceptivos, cognitivos e socioculturais.

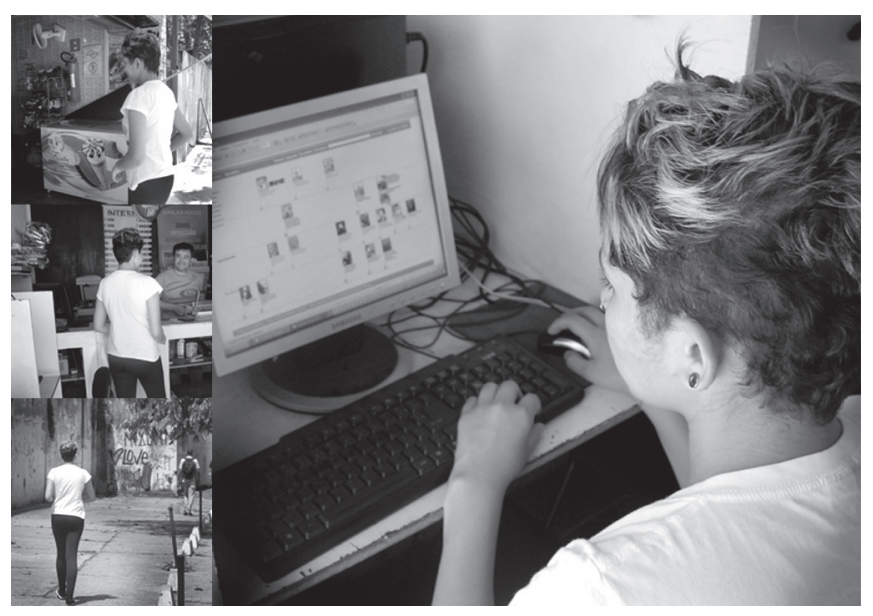

Figura 6: 'Vídeos Conceituais' - Inserção do sistema nas rotinas e cenários de uso da Persona ND.

\section{Considerações Finais}

De acordo com, os estudos do contexto cultural e de uso das interfaces para as cinco regiões definidas, com os resultados obtidos com os testes em campo, com mock-up's, e com os testes simulados de comportamento de uso, com os 'vídeos conceituais', concluiu-se que os 'Indutores de Atividades' das regiões do Brasil, norte, nordeste, centro-oeste, sudeste e sul, possuem aptidões diferentes para a interpretação os elementos visuais, as interfaces, os modos de visualização, os modos de navegação e os modos de interação. O repertório cultural, os cenários de uso e as rotinas de uso, recorrentemente, propiciaram interpretações distintas, nas narrações feitas sobre o mesmo 'fato histórico'. Revelaram também, uma propensão ao estabelecimento na maioria dos casos das mesmas relações, mas com algumas diferenças nas seleções e cruzamentos entre os 'fatos históricos'.

Os resultados apontam a necessidade do estabelecimento de um sistema ubíquo de visualização dinâmica de dados, que seja capaz de adequar-se aos 'Indutores de Atividade', definidos pelos diferentes perfis socioculturais os quais distinguem propensões e limitações. Pois, todos os 'Indutores de Atividade' poderiam estar, com o auxílio de sistemas de visualização dinâmica de dados, capacitados a induzir seleções, análises, interpretações e narrativas, estando familiarizados ou treinados, em certo modo, a utilizar os princípios e as técnicas de manipulação da informação. A prática da usabilidade, com o objetivo de criar interfaces amigáveis e transparentes, exige do designer de sistemas na concepção, desenvolvimento e implantação, atenção com os 
comportamentos, os signos, os símbolos, os artefatos, os valores, os credos, os sonhos e as preferências de cada uma das comunidades. No decorrer da pesquisa, observou-se que existem diferentes níveis de acesso a educação, em cada região, e que mesmo, em regiões com uma formação mais precária, existem 'Indutores de Atividade' com uma capacidade excelente de resposta aos estímulos de um sistema de visualização de dados. Deste modo, o sistema de visualização dinâmica de dados deve ser baseado num 'modelo sociocultural', que considere as diferenças socioculturais regionais, e ao mesmo tempo, as diferentes condições de vida e níveis educacionais, a fim de promover um sistema amigável que aproxime o 'Indutor de Atividades' das informações dispostas, para que seja instigado e capaz de saber selecionar, relacionar, manipular, reorganizar, e que deste modo, esteja sagazmente apto a interpretar e a estabelecer narrativas do seu conhecimento sobre a história do design.

\section{Referências}

Akker, J., Bannan, B., Kelly, A. E., Nieveen, N., \& Plomp, T. (2010). An introduction to educational Design Research. Enschede; NL: SLO.

Bonsiepe, G. (2013). Design, cultura e sociedade. São Paulo, SP: Blucher.

Cardoso, R. (2012). Design para um mundo complexo. São Paulo, SP: Cosac Naify.

Cooper, G. (1998). Research into Cognitive Load Theory and Instructional Design at UNSW. Retrieved from: http://dwb4.unl.edu/Diss/Cooper/UNSW.htm.

Damásio, A. R. (2004). Em busca de Espinosa: prazer e dor na ciência dos sentimentos. São Paulo, SP: Companhia das Letras.

Fulton, S. \& Fulton, J. (2011). HTML5 Canvas: Native Interactivity and Animation for the Web. California CA: O'Reilly.

Geary, D. (2012). Core HTML5 Canvas: Graphics, Animation, and Game Development. Indiana, IN: Prentice Hall.
Gibson, J. J. (1950). Perception of a visual world. Cambridge, UK: The Riverside Press.

Instituto Brasileiro de Geografia e Estatística - IBGE. (2013). Censo 2011 Educação, trabalho e qualidade de vida. Retrieved from: http://www.ibge.gov.br/home/estatistica/populacao/condicaodevida /indicadoresminimos/suppme/default_educacao.shtm.

Miranda, J. B. (1998) Da interactividade: crítica da nova mimesis tecnológica. In: Giannetti, C. (Org.) Telemática: telecomunicação, internet e ciberespaço (pp.119-128). Lisboa, PO: Relógio D’Água.

Nunes, M. P. \& Giraffa, L. M. M. (2003). A educação na ecologia digital. Retrieved from: http://www3.pucrs.br/pucrs/files/uni/poa/facin/pos/relatoriostec/tr 032.pd.

Rosa, J. G. S. \& Moraes, A. (2012a). Avaliação e Projeto no Design de Interfaces. Teresópolis, RJ: 2AB Editora.

Rosa, J. G. S. \& Moraes, A. (2012b). Design Participativo. Rio de Janeiro, RJ: Rio Books.

Rosenfeld, L. \& Morville, P. (2002). Information Architecture for the world wide web. Sebastopol, CA: O’Reilly Media.

Santaella, L. (1998). A percepção: uma teoria semiótica. São Paulo, SP: Editora Experimento.

Santos, L. M. A. \& Tarouco, L. M. R. (2007). A importância do estudo da teoria da carga cognitiva em uma educação tecnológica. Retrieved from: http://seer.ufrgs.br/renote/article/viewFile/14145/8082.

Tufte, E. R. (2001). Envisioning Information. Connecticut, CT: Graphics Press.

Tufte, E. R. (2001). The Visual Display of quantitative information. Cheshire, UK: Graphics Press.

Turban, E., Potter, R. \& Rainer, J. R. K. (2007). Introdução a sistemas de informação: uma abordagem gerencial. São Paulo, SP: Elsevier.

W3schools. (2013). HTML5: geolocation. Retrieved from: http://www.w3schools.com/html/html5_geolocation.asp. 